\title{
Gerhart Hauptmann und die zeitgenössische Psychiatrie im Spiegel seiner Werke
}

\author{
von Klemens Dieckhöfer
}

\section{Zusammenfassung}

Im Euvre des «Dichters des Mitleids» spielen inmitten sozialkritischer Bezüge um das Leid des Menschen psychisch Kranke, Arzte und psychiatrische Einrichtungen eine besondere Rolle. Die Ausstrahlungskraft August Forels und die atmosphärischen Eindrücke vom Zürcher Burghölzli müssen schon 1888 auf Hauptmann prägend gewirkt haben. Die Anstalt Leubus (Schlesien), die Provinzial-Heilanstalt Stralsund und die Einrichtung in Dalldorf bei Berlin bilden weitere Kulissen für Hauptmanns Begegnungen mit dem psychisch Abnormen und seine geradezu untrennbare Affinität zu psychischen Grenzsituationen in seinen Werken.

\section{Einleitung}

Gerhart Hauptmann (1862-1946) ist in die Literaturgeschichte als der «Dichter des Mitleids» eingegangen. Menschliches Leid ${ }^{1}$ wurde immer wieder von ihm in den Mittelpunkt seines dramatischen Euvre gerückt. Ein «stilles Wehgeschrei» ${ }^{2}$ geradezu durchzieht sein gesamtes Werk. Es wundert daher nicht, daß inmitten solcher sozialkritischer Bezüge um das Leid des Menschen psychisch Kranke, aber auch Ärzte und psychiatrische Einrichtungen immer wieder eine besondere Rolle für Hauptmann gespielt haben.

Ohne hier auf die psychologisch einfühlende, erhöhte Sensibilität und Perzeptionskraft der schöpferischen Persönlichkeit des Dichter-Genius im Werk eingehen zu können, wollen wir uns auf die Rezeption der damaligen praktischen Irrenheilkunde durch Gerhart Hauptmann beschränken, mit der der Dichter oft in Berührung kam.

Die Beziehung Gerhart Hauptmanns und seines Bruders Carl (1858-1921) zu dem Zürcher Psychiater August Forel (1848-1931) wurden von Walser ${ }^{3}$ unlängst dargestellt. Dabei stand das Alkoholabstinenzproblem ganz im Mittelpunkt dieser Untersuchung. 


\section{Die psychiatrische Universitätsklinik Zürich-Burghölzli und ihr Einfluß auf Gerhart Hauptmann}

Gerhart Hauptmann geriet im Frühjahr und Sommer 1888 in den Bannkreis Forels, der seit 1879 die Direktion des Zürcher Burghölzli übernommen hatte. Wie Walser ${ }^{4}$ zeigte, gelang dieser Kontakt für die Brüder Hauptmann über den Historiker Arthur Böhtlingk (1849-1929), der als alter Bekannter der Familie Forel Gerharts Mentor an der Universität Jena gewesen war.

Die Monate in der Umgebung des Burghölzli scheinen nun für Gerhart Hauptmann eine erste Quelle ansteckender Faszination für sein Studium der Psychopathologie gewesen zu sein, ein Phänomen, das neben seiner zeitlebens währenden Sehnsucht nach Griechenland als dem idealen Wirkungsraum schöpferischer Dichterphantasie ${ }^{5}$ sein Interesse für die Psyche des Menschen in besonderer Weise zu wecken verstand.

\subsection{Zur Entstehungsgeschichte des Burghölzli}

Die Anstalt Burghölzli hatte ihr Entstehen ursprünglich einem fortschrittlichen Geist jener Epoche zu danken. Kein geringerer nämlich als Wilhelm Griesinger (1817-1868) hatte sich dafür eingesetzt, daß die Planung des damals noch weitgehend verheerenden Irrenwesens auch im Kanton Zürich in neue Bahnen gelenkt wurde. Griesinger war von 1860-1865 Professor für Innere Medizin an der Universität Zürich und ließ sich stets von der Überzeugung leiten, daß Geisteskrankheiten körperlichen Krankheiten gleichzusetzen seien und das «Non-Restraint» absoluten Vorrang in der Behandlung der Kranken haben müsse.

Die Anstalt Burghölzli als kantonale Einrichtung für akute und heilbare Geisteskranke und gleichzeitig als psychiatrische Klinik der Universität Zürich wurde nach endgültigen Beschlüssen des Großen Rates im Jahre 1864 schließlich sechs Jahre später, 1870, bezogen ${ }^{6}$, war also im Jahre 1888, als der 26jährige Enthusiast Gerhart Hauptmann sie als Besucher bei Forel kennenlernte, schon 18 Jahre alt.

\subsection{August Forels Ausstrahlungskraft auf den jungen Dichter Gerhart Hauptmann}

Von Forel bekannte Gerhart Hauptmann: ${ }^{7}$ 
«Er ist es, dessen Erschliessungen von überwiegendem Einfluss auf mich gewesen sind. Er hat mir ein unverlierbares Kapitel von Wissen um die menschliche Psyche vermittelt.»

Gerhart Hauptmann muß von dem Irrenhaus Burghölzli so fasziniert gewesen sein, daß er es mit «schauerlichem Doppelsinn» das «Haus der höchsten Weisheit dieser Welt» nannte und

«auch ausserhalb seiner Mauern bin ich immer mit dem Notizbuch in der Hand, wo ich ging und stand, den psychischen Sonderbarkeiten der Menschen nachgegangen». ${ }^{8}$

Hauptmann hatte durch den engen Kontakt zu Forel insbesondere auch Gelegenheit, an dessen Vorlesungen teilzunehmen. So lernte er «nach und nach alle hauptsächlichen Formen des Irreseins» unterscheiden.

Für den medizinischen Laien war dabei die Krankheitsuneinsichtigkeit einer schizophren Erkrankten mit einem organisierten Wahnsystem (die Patientin war überzeugt, eine natürliche Tochter Kaiser Friedrichs III. zu sein) besonders eindrucksvoll, zumal ein im Kolleg aufgerufener studentischer Praktikant bei der Exploration zunächst nichts Krankhaftes eruieren konnte, so daß dieser zu dem für die Patientin natürlich höchst erfreulichen diagnostischen Urteil kam, daß man bei ihr «von einer Krankheit nichts feststellen» könne. ${ }^{9}$

Hier spätestens rebellierte der Laie Hauptmann gegen das ihm hart erscheinende diagnostische Urteil einer «fixen Idee» als Krankheit.

Sein Argument lautete in fragender Thesenstellung: «... ist hier nicht zwischen Gesundheit und Krankheit nur ein gradueller Unterschied?.» ${ }^{10}$

Anders steht es mit Hauptmanns Beurteilung der Lues:

«Hier steht die wahnhafte Idee des Paralytikers inmitten der Grausigkeit des von Marasmus geprägten körperlich Kranken. Da kommt auch dem Laien kein Zweifel mehr auf, dass dem paralytischen Wahn, ein Herkules zu sein, als Ausfluss einer körperlich begründbaren Geistesstörung Krankheitswert zukommt.» ${ }^{11}$

\subsection{Der bauliche und atmosphärische Zustand des Burghölzli: Reflexionen Gerhart Hauptmanns hierzu}

Der bauliche Zustand der Anstalt Burghölzli war seinerzeit bei Besuch Hauptmanns schon nicht mehr als zeitgemäß zu bezeichnen, da das Gebäude immerhin schon 18 Jahre alt und die Entwicklung diesbezüglich rasant fortgeschritten war. In Rheinpreußen war beispielsweise gerade eine fruchtbare Aufbauperiode von Anstaltsneubauten nach den neuesten Gesichtspunkten einer Non-Restraint-Therapeutik abgeschlossen: Erwähnt seien 
hier die Anstalten Andernach, Düsseldorf-Grafenberg und Merzig/Saar (jeweils 1876 gegründet), Düren (1878) sowie Bonn (1882).

Angesichts dieser in Deutschland und in der Schweiz unterschiedlichen Fortentwicklung der Anstaltsneugründungen und einschlägigen Architektur, die dem Dichter gewiß nicht unbekannt war, muß Hauptmann denn auch von der Unterbringung einer erregten Kranken «in einem Gewölb des Burghölzli» erschüttert gewesen sein:

«Plötzlich kroch sie die Wand hinauf und hing sich an das vergitterte Fenster... Sie lallte von Sonnen, Planeten und anderen Weltkörpern, vielleicht schoss sie selbst, das lebendige Entsetzen, in unendlicher Einsamkeit durch den grundlosen Raum.» ${ }^{12}$

In späteren Jahren ${ }^{13}$ erinnert sich Hauptmann dieser Patientin; in seiner Nachlese zur Autobiographie ${ }^{14}$ heißt es:

«Isoliert in einem kerkerartigen Bau, der nach Raubtierhaus roch, war eine Frau untergebracht. Im höchsten Grade aufgeregt, lief sie umher. Es hiess, sie werde zuweilen tobsüchtig. Einmal stieg sie an der Wand empor und hing oben an einem mit Eisenstangen verwehrten Fenster. Durch dieses Fenster blickte sie, gespannt und berauscht, gegen den Himmel. Solche Kranken werden zuweilen tagelang in warme Bäder gesteckt, um sie zu beruhigen.»

\subsection{Therapeutische Schwerpunkte im damaligen Burghölzli aus der Sicht Gerhart Hauptmanns}

Für zeitgemäße Behandlungsformen war das Burghölzli unter Forel renommiert. Spielten in der wissenschaftlichen Diskussion damals vorwiegend Vererbungsfragen im Zusammenhang mit der Deszendenztheorie und Alkoholabstinenz eine große Rolle ${ }^{15}$, so konzentrierte sich das Interesse der frühen Psychotherapie auf die verschiedenen Formen der Hypnose. Die bravouröse Hypnoanalyse und Suggestionsmethode Forels in einer seiner Kollegstunden gehört zu den glänzendsten Eindrücken von der zeitgenössischen experimentellen Seelenheilkunde, die der junge Gerhart Hauptmann in sich aufnahm ${ }^{16}$.

\section{Hauptmanns dramatisches Fragment «Mutterschaft»; Ort der Handlung: die Anstalt Leubus}

Die Anstalt Leubus in Niederschlesien, die 1830 aus einem im Jahre 1810 säkularisierten Zisterzienserkloster (1906 wurde eine neue Anstalt im 
Ortsteil Leubus II - Städtel - eröffnet) entstand, ist Ort eines dramatischen Fragmentes mit dem Titel «Mutterschaft» ${ }^{17}$, das im April 1905 aus der Feder des Dichters entstanden ist.

Im dritten Akt dieses fragmentarischen Entwurfs beschreibt uns Hauptmann die Insassen der Anstalt. Im Mittelpunkt seiner Betrachtung der Krankheitsbilder standen offenbar wahnbildende Psychosen.

«Jeder von ihnen vertritt ein Wahnsystem und hat seine fixe Idee» ${ }^{18}$, schildert uns Hauptmann.

Die Paranoia-Kranke, die der junge Dichter 1888 im Burghölzli während der Kollegstunden Forels sah, findet sich auch unter den Wahnkranken: «Eine sagt, sie sei die Tochter des Kaisers Friedrich.» ${ }^{19}$

\subsection{Zum Inhalt des Dramas "Mutterschaft»}

Im sozialkritischen Mittelpunkt dieses Dramas wird eine in der Öffentlichkeit bloßgestellte Ärztin geschildert, die sich für eine verzweifelte Mutter mit einem außerehelich empfangenen Kinde einsetzen und zudem ein Mütterheim gründen will.

Unnachgiebiger Protest der Kleinstadt bringt die Praktikerin schliesslich an der Hand eines Irrenarztes in die Paranoia-Abteilung von Leubus. Der soziale Konflikt ist aber nach Überzeugung der Ärztin vorprogrammiert. Die Schmach sei schlimmer als der Tod, hieraus resultiere die Notwendigkeit der Abtreibung, Quintessenz: «Ihr [Irrenärzte] seid Mörder, nicht solche Frauen sind Mörder.»

Diese Idee ist aber für die damals herrschende Psychiatrie die krankmachende Wahnidee, die die Ärztin in den Gewahrsam der geschlossenen Anstalt Leubus gebracht hat, so will es die dichterische Freiheit.

Im Sinne von Kurt Schneider ${ }^{20}$ verlangt mithin das Dichtwerk hier in unnaturalistischer Weise den durchgehenden Motivzusammenhang und nicht ein Abreissen der Verständlichkeit als Ausdruck einer inhaltlich wahnhaft verfälschten Vorstellung. Das aussereheliche Kind wird als unmoralisch gebrandmarkt, so will es die Theorie des Anstaltsdirektors. Die Moral solle triumphieren. Um sich nicht enterben zu lassen, leugnet schliesslich die Ärztin ihre als unsinnig gebrandmarkten Ideen. Eine Liebesbeziehung zwischen Anstaltsdirektor und bekehrter Ärztin beschleunigt dabei die Entlassung in die Freiheit. Drei Jahre später will ein Gericht den Gesundheitszustand der Ärztin, die inzwischen entmündigt und nach dem Tod ihres Vaters enterbt wurde, prüfen. Auf Empfehlung ihres Verteidigers wird zunächst ein Loblied auf die herrschende Moral gesungen, doch dann bricht es aus der Ärztin heraus:

«Entmündigt mich, ich lache euch aus: Ich lache die ganze Stadt aus; denn die Gesellschaft ist feige, vor dem Mutigen weicht sie zurück.» ${ }^{21}$

Das Gericht, ratlos und konsterniert zunächst, lässt sich erst durch das Erscheinen des gebrandmarkten Kindes, ihres Sohnes, eines zweieinhalbjährigen prächtigen Knaben, der 
ohne Vater unehelich bei der Mutter aufwuchs, umstimmen, so dass die Enterbung schliesslich abgewendet werden kann.

Hauptmann begründet in abschließenden «Notizen» ${ }^{22}$ die dichterische Notwendigkeit dieser unnaturalistischen dramatischen Entwicklung mit den Worten:

«Ein Mensch ohne irgendeinen kleinen Wahnsinn lebt nicht und hat nichts Göttliches.»

\section{Hauptmanns Urteil über die Psychiatrie nach seinem Besuch in den Anstalten des Regierungsbezirks Stralsund}

In seiner Nachlese zur Autobiographie ${ }^{23}$ gibt uns Hauptmann ein weitgehend enttäuschendes Résumé vom Zustand der Psychiatrie nach dem Ersten Weltkrieg. Nach seinen eigenen Worten besuchte Hauptmann damals - also Anfang der 20er Jahre - «die großen Irrenanstalten des Regierungsbezirks Stralsund mit dessen Residenten». ${ }^{24}$

Die Stadt Stralsund in der Provinz Pommern verfügte schon seit 1912 über eine Provinzial-Heilanstalt mit ca. 1200 Betten, auch über eine forensische Abteilung, ein sog. «festes Haus», nachdem die Provinzial-Irrenanstalt für Unheilbare im März 1900 geschlossen worden war und ihre Insassen nach Treptow a/Rega verlegt worden waren.

Weil Hauptmann ausdrücklich «die» großen Irrenanstalten des Reg.-Bezirks Stralsund erwähnt, dürfte unzweifelhaft sein, daß zwar mehrere Einrichtungen in sein Blickfeld traten. Dieser Regierungsbezirk war indes nur bis zum Jahre 1932 eigenständig und wurde dann dem Regierungsbezirk Stettin zugeschlagen; es finden sich geographisch hier s.Zt. (Hauptmanns Bericht datiert zwar von 1937, erwähnt aber eigens ausschließlich eben nur den Regierungsbezirk Stralsund) insofern nur zwei Einrichtungen: Die Psychiatrische und Nervenklinik der Universität Greifswald (Gründungsjahr 1889) mit 135 Betten (Stand 1937) sowie die Provinzialheilanstalt der Stadt Stralsund selbst (gegr. 1912) mit 1217 Plätzen (+ forensische Abteilung, sog. festes Haus für 50 psychisch kranke Rechtsbrecher, Stand 1937).

Der Besuch Hauptmanns (zusammen mit seinem Sohn Benvenuto) kann eigentlich nur der Provinzial-Heilanstalt der Stadt Stralsund selbst gegolten haben («der weite Häuser- und Gartenkomplex»). Hier stand nicht der bauliche Zustand im Kontext mit dem psychisch Kranken im Mittelpunkt 
der Betrachtung des Dichters, sondern der kranke Mensch selbst, der die beiden Besucher bis zum Weinen erschütterte.

Beschrieben wird ein schizophrener Kranker, dessen Stereotypien («röhrende» Laute) sich nur durch ein kurzes Gespräch und anschließendes zauberhaftes Geigenspiel des Kranken vor den tief bewegten Besuchern unterbrechen ließ, zumal sich hierbei erwies, daß der Patient «ein guter Orchesterspieler war und zuletzt im Kurorchester zu Interlaken gewirkt hatte». ${ }^{25}$ Nach Ende des Geigenspiels setzten wieder diese Schaudern hervorrufenden sprachlichen Stereotypien, sog. Verbigerationen, ein. Hauptmann dachte sich, daß dieser Mann vielleicht als Musiklehrer der Anstalt oder Leiter eines Quartetts oder kleinen Orchesters tätig werden könnte, zweifelte indes, ob eine solche therapeutische Maßnahme in dieser Anstalt überhaupt gelingen könnte:

«Das aber setzt eben Liebe zur Kunst, zur Musik voraus und die Ehrfurcht vor dieser göttlichen Gabe. Ob sie bei dem Leiter dieser Anstalt vorhanden war, weiss ich nicht.» ${ }^{26}$

Für Hauptmann schien es wohl nahe zu liegen, daß ein Anstaltsleiter sich u. a. auch offen zeigen sollte für Musiktherapie und andere Verfahren, die der Rehabilitation eines psychisch Kranken dienen können, beim Therapeuten jedoch ein besonderes künstlerisches Einfühlungsvermögen voraussetzen. Im vorliegenden Fall schien der Dichter dies wohl zu bezweifeln. Solche leise gehegten Zweifel an der Fähigkeit der Irrenärzte zu der an sich möglichen, sinnvollen Rehabilitation ihrer Patienten bringt Hauptmann an anderer Stelle in den «Annalen» überaus deutlich und ungeschminkt zum Ausdruck $^{27}$ :

«Durch den Geist wurde auf Geisteskranke damals nicht eingewirkt», ferner: «Der Psychiater von heute hat zum Menschen ein nur sachliches, ganz unpersönliches Verhältnis. Was ausser seiner Krankheit an ihm ist, interessiert ihn nicht. In dieser Beziehung und hiergegen hat selbst die Behandlung der Besessenen durch den Priester einen guten Kern»; und schliesslich: «Die geistige Therapie in den Irrenhäusern von heut und auch die sonstige geistige Pflege von Kranken ist gleich Null.»

Eine derartige Steigerung herber Kritik und scharfer Vorwürfe gegenüber der zeitgenössischen Psychiatrie der 20er Jahre wird für Hauptmann auch durch die ihm bekannte Freudsche Analyse und Psychotherapie nicht gemildert: Einer solchen Therapeutik wirft Hauptmann «gefährliche geistige Sektion» und ... «künstlich herbeigeführte Geisteserkrankung» vor ${ }^{28}$. 


\section{Hauptmanns Reime auf den «Blechschmied» von Dalldorf}

In den nachgelassenen Werken und Fragmenten ${ }^{29}$ findet sich eine Sammlung von 22 Distichen, die mit der Überschrift «Blechschmied» versehen und im März 1922 entstanden sind. Im 2. Verspaar ist dabei von Dalldorf die Rede.

Die Einrichtung in Dalldorf wurde nach verschiedenen einschlägigen kleineren Vorläufern seit dem Anfang des 18. Jahrhunderts als «Irrenanstalt der Stadt Berlin» im Regierungsbezirk Potsdam am 20.1.1880 gegründet. Um die Jahrhundertwende wurden dort nahezu 2600 Patienten verpflegt. Die Anstalt mit einer Vielzahl von sog. Filialen im Raum Berlin zwischen Charlottenburg und Steglitz gehörte damit (neben Bedburg-Hau, Gründung 1911) zu den grössten ihrer Art im Gebiet des Deutschen Reiches.

Der Blechschmied, eingekerkert in einer dumpfen Zelle, ist für Hauptmann Symbol des unheilbar Geisteskranken:

«Ganz unheilbar, so sagt der Arzt, sind solcherlei Kranke, nun gar dieser, nie war hoffnungsloser ein Fall.» ${ }^{30}$

Zwangsjacke und Fessel können nicht hindern, daß der seltsame Zelleninsasse mit uneinfühlbarer Wucht den Hammer schwingt; mehr als fragwürdig bleibt dabei, ob hier überhaupt produktiv etwas geleistet wird. Hauptmann schildert vielmehr mit der Hoffnungslosigkeit eines solchen Daseins die Sinnlosigkeit des Soseins dieses Hammerschwingers und nur scheinbaren Blechschmieds. Denn der schizophrene Wahn nimmt im Stumpfsinn der traurigen Zelle, die von «Kloakenarom» parfümiert ist, armselige Gestalt an in der sog. doppelten Orientierung, ähnlich wie dies bei dem Lizentiaten Vidriera von Cervantes ${ }^{31}$ der Fall ist:

«Nein, er ist gar kein Schmied: er hält sich nur selbst für den Blechschmied, ein armseliger Narr dünkt sich der König zu sein.» ${ }^{32}$

Stumpfheit, affektive Verödung und Stereotypien mit motorischem Leerlauf in Form der hämmernden Automatismen bestimmen das Bild eines offenbar Defektschizophrenen. In diesem «Gipfel des Stumpfsinns» ${ }^{33}$ kommt es aber auch gelegentlich zu hyperkinetischen Erregungszuständen und Impulshandlungen, wie der Dichter aufmerksam beobachtet hat:

«Sei indes auf der Hut, du begegnest des Nachts wohl dem Blechschmied, der sein eignes Gebirg hilfeschreiend durchirrt.» ${ }^{34}$ 


\section{Schlußbemerkung}

Die Bemerkung Diltheys ${ }^{35}$, daß die Phantasie ihre Gestaltungen aus der äußeren und inneren Erfahrung aufbaut, trifft in eminenter Weise für G.Hauptmanns Auseinandersetzung mit dem psychisch Abnormen und seiner Repräsentanz in der Umwelt des Dichters zu.

Die frühen Begegnungen mit dem psychisch Kranken in den damaligen großen Anstalten prägten sich dem feinfühligen Psychopathologen aus Naturtalent tief ein. Hauptmanns ganzes Werk ist von einer geradezu untrennbaren Affinität zu psychischen Grenzsituationen gekennzeichnet.

Herbe Kritik des Nicht-Arztes ${ }^{36}$ an den gewachsenen Einrichtungen der Irrenheilkunde, die in Hauptmanns Jugend erst zur wissenschaftlichen Psychiatrie heranreifte, hinderte den Schriftsteller nicht, das psychisch Abnorme in allen Schattierungen, und dies inmitten der damaligen «Ballungszentren» der psychisch Kranken der «Heilanstalten», als dramatische Figuren $^{37}$ zu wählen.

\section{Anmerkungen}

\section{Vorbemerkung:}

Für die Zitationen zu G. Hauptmann wurde stets die Centenar-Ausgabe (= CA) benutzt: Gerhart Hauptmann. Sämtliche Werke. Hrsgeg. v. H.E.Haß. Fortgeführt v. M. Machatzke. Propyläen, Frankfurt a. M./Berlin 1962 ff. Die im Text gelieferten Hinweise zu den Anstalten sind entnommen den einschlägigen Werken von Laehr, H., Die Anstalten für Geisteskranke ..., de Gruyter, Berlin/Leipzig 1937 sowie Guttstadt, A., Krankenhaus = Lexikon für das Deutsche Reich, Berlin, Reimer 1900.

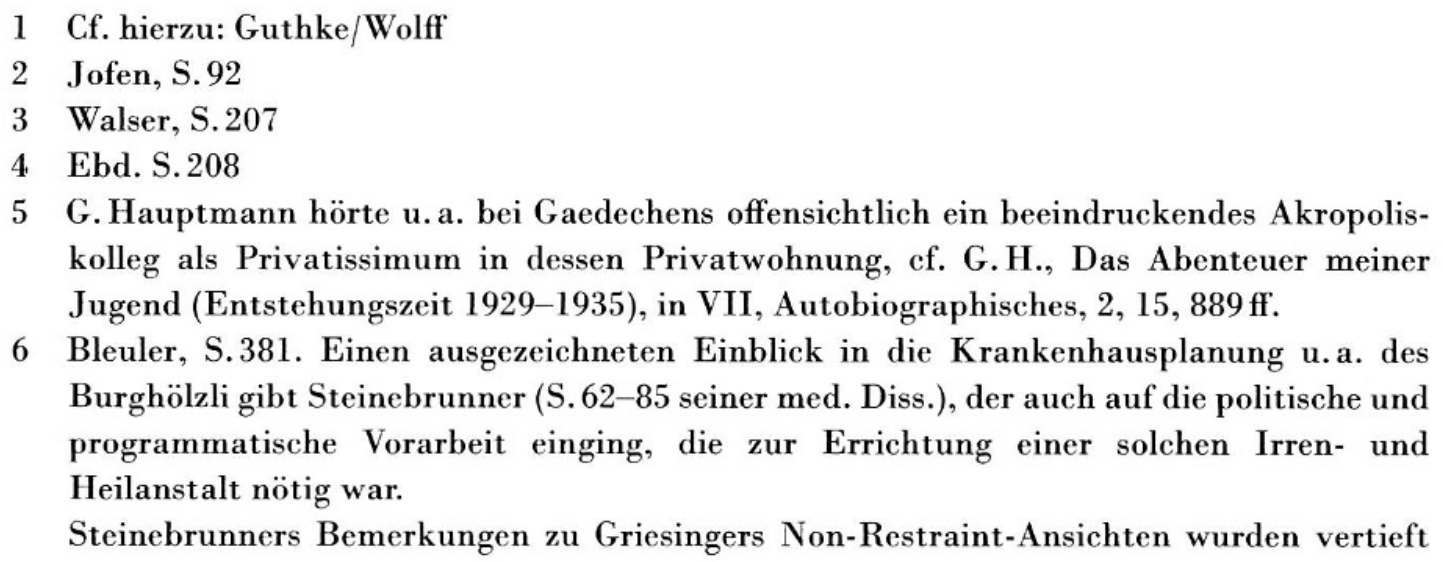
kolleg als Privatissimum in dessen Privatwohnung, cf. G.H., Das Abenteuer meiner Jugend (Entstehungszeit 1929-1935), in VII, Autobiographisches, 2, 15, $889 \mathrm{ff}$.

6 Bleuler, S.381. Einen ausgezeichneten Einblick in die Krankenhausplanung u.a. des Burghölzli gibt Steinebrunner (S.62-85 seiner med. Diss.), der auch auf die politische und programmatische Vorarbeit einging, die zur Errichtung einer solchen Irren- und Heilanstalt nötig war.

Steinebrunners Bemerkungen zu Griesingers Non-Restraint-Ansichten wurden vertieft 
dargestellt in der von Cordula Geduldig angefertigten med. Dissertation, die die Rezeption dieser neuartigen Behandlungsmethode im deutschen Sprachraum berücksichtigt.

7 So in: G. H., Das Abenteuer meiner Jugend, in CA, VII, Autobiographisches, 2, 39, 1057

8 Ebd. 2, 39, 1058 f.

9 Ebd. 2, 40, 1063

10 Ebd.

11 Ebd. 2, 39, 1064

12 Ebd. 2, 39, 1065. - Erst 1903 und 1909 wurde je ein Pavillon für akut behandlungs- und pflegebedürftige Kranke auf der Männer- und Frauenseite hinzugefügt (cf. Bleuler, a.a.O., S.382).

13 Entstehungszeit 25./26.3.1937, also mindestens 2 Jahre später als «Das Abenteuer meiner Jugend», cf. Anm. 5

14 G. H. Nachlese zur Autobiographie. 2. Vierteljahrhundert. Paralip I. zu «Annalen` in: CA XI, 544

15 Einen guten Überblick über die damalige therapeutische Situation geben A. Eulenberg, Real-Encyclopädie der gesammten Heilkunde. Urban \& Schwarzenberg, Wien und Leipzig 1896; Kapitel Irrenbehandlung, S.671 und der Bonner C.Pelman: Über die Behandlung der Geisteskranken, in: Die Deutsche Klinik, hrsg. von E.v.Leyden u. F. Klemperer, Urban \& Schwarzenberg, Berlin-Wien, 1906, cf. hier besonders S. 1088 und 193

16 G.H., Das Abenteuer meiner Jugend, in C.A. VII Autobiographisches, 2, 40, 1066

17 G.H. Mutterschaft, in: C.A. IX Nachgelassene Werke, Fragmente, S. 297 ff.

18 Ebd., S.298

19 Ebd.

20 Schneider, K., S.13

21 G. H., Mutterschaft, in CA IX Nachgelassene Werke, Fragmente, S. 300

22 Ebd., S.301

23 G. H. Paralip. I. zu 〈Annalen`, in: C.A. XI, S. 541 ff.

24 Ebd., S. 546

25 Ebd.

26 Ebd.

27 Ebd.

28 Ebd.

29 G. H., Blechschmied, in CA XI, Nachgelassene Werke, Fragmente, S. $673 \mathrm{ff}$.

30 Ebd., S. 674, 3

31 Dieckhöfer, cf. Cervantes, Der Lizentiat Vidriera

32 G.H., Blechschmied, a. a. O., S. 674, 7

33 Ebd., S. 675, 18

34. Ebd., S. 65, 19

35 Dilthey, zit. n. K. Lange-Eichbaum, W. Kurth, Genie, Irrsinn und Ruhm, S. 105

36 K. Schneiders Feststellung bestätigt sich damit: «... Nach dem, was wir hörten, wissen wir (= Ärzte), dass wir nicht viel Liebes erwarten dürfen, und in gewisser Beziehung haben wir es auch nicht verdient» (cf. K. Schneider, a. a. O., S.13). - In den meisten der Hauptmannschen Werke finden sich übrigens Ärzte als wichtige Figuren der dramati- 
schen Handlung. Andererseits bekennt Hauptmann noch ein Jahr vor seinem Tode auf dem Wiesenstein in Agnetendorf am 5.4.1945:

«Ich selber wurde unter Ärzten nicht klar, obgleich ich auch einer von ihnen war.»

Cf. G. H., Nachlese zur Lyrik 1933-1946, in C.A. XI, Nachgelassene Werke, Fragmente, S. 748

37 Hier sei erinnert an Lope de Vega: «Die Irren von Valencia», cf. Dieckhöfer, K.: Lope de Vegas Komödie...

\section{Literaturverzeichnis}

Bleuler, M.: Geschichte des Burghölzlis und der psychiatrischen Universitätsklinik, in: Zürcher Spitalgeschichte, hrsg. vom Regierungsrat des Kantons Zürich. Zürich 1951

Dieckhöfer, K.: Der Lizentiat Vidrera. Ein Beitrag zur Psychopathologie künstlerischen Schaffens bei Cervantes. Schweiz. Arch. Neurol. Psychiat. 114 (1974), 357-365

Dieckhöfer, K.: Lope de Vegas Komödie «Los Locos de Valencia) - ein kulturhistorischer Einblick in eine psychiatrische Anstalt des 15. Jahrhunderts. Schweiz. Arch. Neurol. Psychiat. 116 (1975), 343-351

Dilthey, W.: Über die Einbildungskraft der Dichter. Zschr. Völkerpsychol. u. Sprachwissenschaft. Bd.10. Dümmler, Berlin 1878, Zit. n. Lange-Eichbaum, W., W.Kurth, Genie, Irrsinn und Ruhm. 6. Aufl., Reinhardt, München/Basel 1967

Geduldig, C.: Die Behandlung von Geisteskranken ohne physischen Zwang. Die Rezeption des Non-Restraint im deutschen Sprachgebiet. Diss. med. Nr. 164, Medizinhistorisches Institut, Zürich 1975.

Guthke, K.S., Wolff, H.M.: Das Leid im Werke Gerhart Hauptmanns. Francke, Bern 1958

Jofen, J.: Das letzte Geheimnis. Francke, Bern, 1972

Schneider, K.: Der Dichter und der Psychopathologe. Rheinland, Köln 1922

Steinebrunner, W.F.: Zwei Zürcher Krankenhausplanungen des 19.Jahrhunderts - ihre ärztlichen Experten, ihre Vorbilder. Diss.med. Nr.129, Medizinhistorisches Institut, Zürich 1971.

Walser, H. H.: Ist das Wohl des Landesherrn in Wein zu trinken? Gesnerus 38 (1981), 207-214. 


\section{Summary}

\section{Gerhart Hauptmann and contemporary psychiatry as reflected in his works}

In the work of Hauptmann, the "Poet of compassion", the mentally ill, doctors and psychiatric institutions play a special role in the context of social criticism concerning human suffering. The charisma of August Forel and the impressions of atmosphere at the Zurich Burghölzli must already have had a formative effect on Hauptmann in 1888. The Leubus Institution (Silesia), the provincial psychiatric hospital in Stralsund and the psychiatric hospital in Dalldorf/Berlin are further backdrops for Hauptmann's encounters with the psychologically abnormal and his almost characteristic affinity for borderline psychological situations in his books.

\section{Résumé}

Gerhart Hauptmann et la psychiatrie contemporaine dans le miroir de ses ouvres

Dans l'œuvre du "poète de la compassion», malades mentaux, médecins et institutions psychiatriques jouent un rôle particulier dans le contexte de la critique sociale face à la souffrance humaine. Le rayonnement d'Auguste Forel et l'atmosphère du Burghölzli de Zurich doivent en 1888 déjà avoir profondément marqué Hauptmann. L'institution Leubus en Silésie, l'hôpital régional de Stralsund et la clinique de Dalldorf/Berlin constituent ensuite les décors successifs dans lesquels Hauptmann rencontre l'anormalité psychique qui fonde dans son œuvre cette affinité profonde avec les situations psychologiques critiques.

Prof. Dr. med. Klemens Dieckhöfer

Medizinhistorisches Institut der Universität

Sigmund-Freud-Straße 25

D-5300 Bonn 1 (Venusberg) 